\title{
Mathematics
}

\section{Hilbert's sixteenth problem}

\section{Ian Stewart}

IN 1900 David Hilbert delivered a celebrated lecture before the International Congress of Mathematicians in Paris. "The close of a great epoch," he said, "not only invites us to look back into the past but also directs our thoughts to the unknown future." Hilbert emphasized the importance of specific problems in mathematical research, and listed 23 problems which seemed to him to be of fundamental interest. It is a measure of the maturity of today's mathematics that most of Hilbert's problems now have answers, but some remain stubbornly intractable, the most notorious being the Riemann hypothesis. The sixteenth of Hilbert's problems comes in two similar but largely unrelated parts: the first asks how the different branches of a plane curve can be arranged; and the second raises the analogous problem of the number of limit cycles arising from a differential equation in the plane. There are some important recent developments, not all positive, on this second part of Hilbert's sixteenth problem.

Before describing the current state of play, I will look at the origins of the problem in more detail. In the late nineteenth century, mathematicians were starting to take a serious interest in nonlinear differential equations. In particular they worked on differential equations of the form $\mathrm{d} y / \mathrm{d} x=Y / X$, where $X$ and $Y$ are polynomials in $x$ and $y$. In general it is not possible to write down explicit solutions to such equations, but Henri Poincaré discovered that a great deal of information can be obtained by topological means. Rewrite the equation as a system $\mathrm{d} x / \mathrm{d} t=X, \mathrm{~d} y / \mathrm{d} t=$ $Y$, where $t$ is a new variable, thought of as representing time. As $t$ varies, the solution $(x(t), y(t))$ describes a curve in the plane. This curve depends on the initial conditions $(x(0), y(0))$, and by considering all possible initial conditions one obtains a system of curves called the phase portrait of the equation. The phase portrait gives a geometrical picture of all possible solutions to the differential equation, and is a powerful tool for a qualitative study.

In a fundamental re-direction of research on differential equations, Poincaré determined the basic topological properties of such phase portraits. In particular he recognized the importance of limit cycles, which occur when a curve in the phase portrait closes up on itself forming a loop. A limit cycle corresponds to a periodic solution of the differential equation, in which the system repeats the same behaviour over and over again, and hence has genuine physical interest. (In practice, some extra technical requirements are imposed when defining limit cycles, but I will omit these here.)

The second part of Hilbert's sixteenth problem asks how many such limit cycles can occur, for polynomials $X$ and $Y$ of given degree. When Hilbert raised the question, it was not even known whether the number of limit cycles must be finite. This weaker version of Hilbert's problem appeared to be solved affirmatively when H. Dulac wrote a lengthy memoir (Bulletin de la Société Mathématique de France 51, 45-188; 1923). The crucial step in Dulac's proof is to show that an infinite

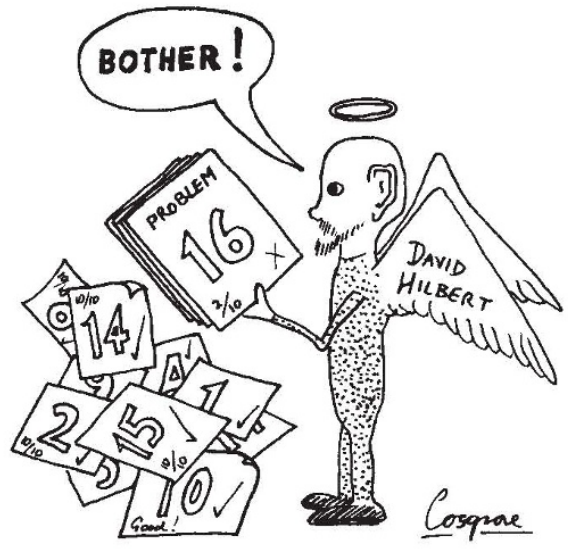

number of limit cycles cannot accumulate around a single point of the phase portrait.

Some time later, Yu.S.Il'yashenko of Moscow State University (Uspekhi Mat. Nauk. 37, 127; 1982) noticed an error in one of the lemmas used by Dulac to establish his main theorem. Now, Il'yashenko has managed to repair some of the results in Dulac's memoir and to prove the finiteness of the number of limit cycles under more stringent conditions. In particular he shows that for polynomials $X, Y$ of degree two, the number of limit cycles occurring in any finite portion of the plane is finite; and that for "almost all" polynomials of degree two, the number of limit cycles in the whole plane is finite. The proofs use modern developments in singularity theory and the theory of normal forms.

A proof of finiteness of the number of limit cycles for polynomials of degree higher than two would be a major step towards solving Hilbert's sixteenth problem. But Hilbert asks for more: namely, the exact number. To be precise, let $H(n)$ be the maximum number of limit cycles that can occur when $X$ and $Y$ have degree $n$. The known results, described above, cannot even show that $H(n)$ is finite. However, in all known examples $H(n)$ is not just finite, but fairly small. The gap between what is conjectured that exists and what is known is enormous.

N.N. Bautin (Mat. Sbornik 30, 181 $196 ; 1952$ ) found a pair of polynomials $X$ and $Y$ of degree two, for which three limit cycles occur. Thus $H(2)$ is at least 3 . This result was bettered by Shi Sonling (Scient. Sinica 23, 153-158; 1980) who found an example with four limit cycles. Thus $H(2)$ is at least 4. This example is a small (and very complicated) perturbation of the system given by $X=-y-10 x^{2}+5 x y+y^{2}$, $Y=x+x^{2}-25 x y$.

The best results to date are that $H(n)$ is at least $1 / 2\left(n^{2}+5 n-14\right)$ for even $n \geqslant 4$, at least $1 / 2\left(n^{2}+5 n-26\right)$ for odd $n \geqslant 9$, and that $H(3) \geqslant 5, H(5) \geqslant 14, H(7) \geqslant 27$. The first two results here are due to N.F. Otrokov (Mat. Sbornik 34, 127-144; 1954). That for $H(3)$ is due to K.S. Sibirskii (Different. Uravneniya 1, 53-66; 1965) and Shi Sonling (Acta Math. Sinica 4, 300$304 ; 1975)$; the last two follow from a general result of Il'yashenko (Mat. Sbornik 78, 360-373; 1969).

Nonlinear differential equations are important in all sciences and are at the forefront of some of the most active areas of current mathematical research. Today's methods for analysing nonlinear differential equations give an excellent understanding of many general phenomena. Moreover, numerical methods, implemented on a computer, can provide very detailed information on any specific differential equation of interest. Hilbert's sixteenth problem falls neatly in between. It concerns a class of equations (those defined by polynomials $X$ and $Y$ of degree $n$ ) which is too restricted to be amenable to general methods, yet too broad (having too many free parameters) to yield to numerical analysis. Additionally, Hilbert's problem asks for global information on the entire phase portrait, whereas many of the most powerful general results are only local.

Mathematicians tend to think of differential equations in two variables as being trivial. This is perhaps true of specific equations, or of the local behaviour of arbitrary equations. The status of Hilbert's sixteenth problem illustrates that the global behaviour of multi-parameter families of equations continues to pose almost insuperable problems. We can sympathize with Hilbert's opening words of 1900: "Who of us would not be glad to lift the veil behind which the future lies hidden; to cast a glance at the next advances of our science and at the secrets of its development during future centuries? What particular goals will there be toward which the leading mathematical spirits of coming generations will strive? What new methods and new facts in the wide and rich field of mathematical thought will the new centuries disclose?"

Ian Stewart is in the Mathematics Institute, University of Warwick, Coventry CV4 7AL, UK. 